\title{
Attosecond pulse walk-off in high-order harmonic generation
}

\author{
D. Kroon, ${ }^{1, *}$ D. Guénot, ${ }^{1}$ M. Kotur, ${ }^{1}$ E. Balogh,${ }^{2}$ E. W. Larsen, ${ }^{1}$ C. M. Heyl, ${ }^{1}$ M. Miranda, ${ }^{1}$ M. Gisselbrecht, ${ }^{1}$ \\ J. Mauritsson, ${ }^{1}$ P. Johnsson, ${ }^{1}$ K. Varjú, ${ }^{2}$ A. L'Huillier, ${ }^{1}$ and C. L. Arnold ${ }^{1}$ \\ ${ }^{1}$ Department of Physics, Lund University, P.O. Box 118, 22100 Lund, Sweden \\ ${ }^{2}$ Department of Optics and Quantum Electronics, University of Szeged, Dóm tér 9, 6720 Szeged, Hungary \\ ${ }^{*}$ Corresponding author: david.kroon@fysik.lth.se
}

Received January 8, 2014; revised March 5, 2014; accepted March 6, 2014;

posted March 7, 2014 (Doc. ID 204087); published March 31, 2014

\begin{abstract}
We study the influence of the generation conditions on the group delay of attosecond pulses in high-order harmonic generation in gases. The group delay relative to the fundamental field is found to decrease with increasing gas pressure in the generation cell, reflecting a temporal walk-off due to the dispersive properties of the nonlinear medium. This effect is well reproduced using an on-axis phase-matching model of high-order harmonic generation in an absorbing gas. @ 2014 Optical Society of America

OCIS codes: (320.7110) Ultrafast nonlinear optics; (020.2649) Strong field laser physics; (020.4180) Multiphoton processes.

http://dx.doi.org/10.1364/OL.39.002218
\end{abstract}

The nonlinear interaction between a focused high-power laser beam and a gas target generates broadband light bursts emitted once every half cycle of the driving field $[1,2]$. In the spectral domain, the half-cycle periodicity and symmetry properties of the target gas result in peaks centered at the odd-order harmonics of the driving pulse carrier frequency. The large bandwidth combined with the coherence of the process, inherited from the generating field, yields pulses with a time duration on the attosecond scale $[\underline{3}, \underline{4}]$. The intrinsic synchronization of the attosecond pulses with the generating field []ㅡ allows for pump-probe experiments on an ultrafast time scale.

By cross correlating the attosecond pulse train (APT) with a weak copy of the generating pulse in a detection gas, while monitoring the generated photoelectron spectrum, the phase difference between consecutive harmonic orders can be extracted [5]. Combined with a measurement of the relative spectral amplitudes, this information allows for a reconstruction of the average time structure of the pulses in the train. This is the well-known RABITT scheme (reconstruction of attosecond beating by interference of two-photon transition) for characterization of APTs.

This technique has recently gained a lot of interest since the comparison of RABITT measurements in different systems, for example, different atomic shells, allows for the determination of their relative photoionization delays [6]. These experiments demand high interferometric stability, on the order of tens of attoseconds, and require control of the timing of the attosecond pulses relative to the fundamental field. In some early implementations of the RABITT scheme $[\underline{5}, \underline{7}, \underline{8}]$, the probe pulse and the generation pulse were both propagated through the generation medium, making it possible to encode the phase relation between the generating and the probing field into the recorded electron spectrogram, but at the same time perturbing the regularity of the pulse train.

In this work, we perform RABITT measurements using an actively stabilized interferometer. We present an experimental study of the group delay of the attosecond pulses relative to the generating field as a function of the gas density in the generation cell. Our results show that the detected pulse train advances by almost 200 as, relative to the fundamental field, as the pressure is increased by a factor of three. This observation is interpreted as a temporal walk-off of the attosecond pulses due to dispersion in the medium and simulated using an on-axis phase-matching model.

The experiments were performed with a Ti:Sapphire femtosecond laser emitting pulses with $20 \mathrm{fs}$ (FWHM) duration, centered around $800 \mathrm{~nm}$, with $1 \mathrm{kHz}$ repetition rate and a pulse energy of $3 \mathrm{~mJ}$. A beam splitter divides the laser output into the probe and the pump arm of a Mach-Zehnder interferometer [see Fig. 1(a)]. The pump pulse is focused into a $6 \mathrm{~mm}$ long cylindrical cell by a $45 \mathrm{~cm}$ focal length parabolic mirror. A pulsed gas valve, synchronized with the laser pulses, releases Ar gas into the cell where the XUV light is generated. The instantaneous gas pressure in the cell is stabilized by monitoring the background pressure in the chamber and controlling the valve opening with a feedback loop. The actual pressure in the generation cell is unknown and is assumed to scale linearly with the background pressure. A $200 \mathrm{~nm}$ thick aluminium filter blocks the fundamental radiation and acts as an amplitude and phase filter for the harmonic radiation. The probe arm and the generation arm of the interferometer are recombined using a holey

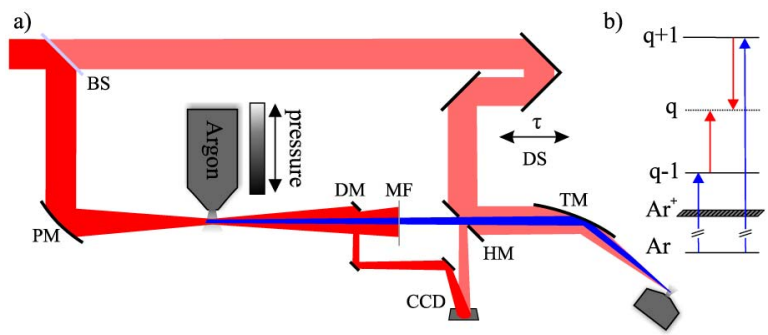

Fig. 1. Schematic outline of (a) the experimental setup and (b) the principle of the measurement. The optical elements are a beam splitter (BS), a parabolic mirror (PM), a d-shaped mirror (DM), a metallic filter (MF), a holey mirror (HM), a toroidal mirror (TM), and a delay stage (DS). 
mirror, which transmits the XUV APT and reflects the outer portion of the probe beam. A gold coated toroidal mirror focuses both beams into the sensitive region of a magnetic bottle electron spectrometer, where an effusive gas jet provides argon as the detection gas.

The delay is monitored by forming spatial interference fringes between the generation beam and probe beam on the CCD chip of a camera. For this purpose, a small portion of the generation beam is split off with a d-shaped mirror prior to the metallic filter; for the probe beam the portion going through the hole in the recombination mirror is used. A computer program extracts the phase of the spatial fringes and sends a feedback signal to a piezoelectric delay stage in the probe arm. Any perturbation of the optical path length introduced in either of the interferometer arms is, thus, actively compensated for. Furthermore, laser beam-pointing drifts, which could be interpreted as a relative length change of the interferometer arms, are avoided by active beam-pointing stabilization before the interferometer. The precision of the delay stabilization was tested in an out-of-loop measurement. A comparison between the active delay stabilization being turned off and on shows that the standard deviation of the extracted phase delay between the pump and probe pulses drops from 200 as to 50 as, for a measurement taken over $400 \mathrm{~s}$.

The experiment consisted of recording spectrograms of the photoelectron energy as a function of the delay $(\tau)$ between the APTs and the infrared (IR) probe field. Sidebands appear between the harmonic peaks in the photoelectron spectrogram due to absorption of a harmonic $(q-1)$ and an IR photon, or by absorption of the next harmonic $(q+1)$ and emission of an IR photon [see Fig. 1(b)]. The sideband intensity oscillates as a function of delay [3] , according to

$$
S(\tau, q)=A+B \cos \left[2 \omega\left(\tau-\tau_{q}\right)\right],
$$

where $A$ and $B$ are quantities that depend on the probability amplitudes of the two processes leading to the same final state and $\omega$ is the fundamental angular frequency. The quantity extracted from the measurement, $\tau_{q}$, is a time offset equal to

$$
\tau_{q}=\left.\frac{\phi_{q+1}-\phi_{q-1}}{2 \omega} \approx \frac{\partial \phi_{\Omega}}{\partial \Omega}\right|_{\Omega=q \omega},
$$

where $\phi_{q+1}$ and $\phi_{q-1}$ are the accumulated phases along the two quantum paths shown in Fig. $\underline{1(b)} . \phi_{q+1}$ is the sum of the phase of the $(q+1)$ th harmonic field and the phase accumulated in the two-photon ionization process []. $\tau_{q}$ can thus generally be written as a sum of two terms, one representing the group delay of the average attosecond pulse in the train and a second term originating from the electronic transition. On the righthand side of Eq. (2), the finite difference is approximated as the derivative of the phase with respect to the angular frequency, $\Omega=q \omega$.

Figure $\underline{2}$ summarizes the experimental results presented in this Letter. Figure 2(a) shows photoelectron spectra obtained at four different pressures in the harmonic generation cell. Each of the spectra has been
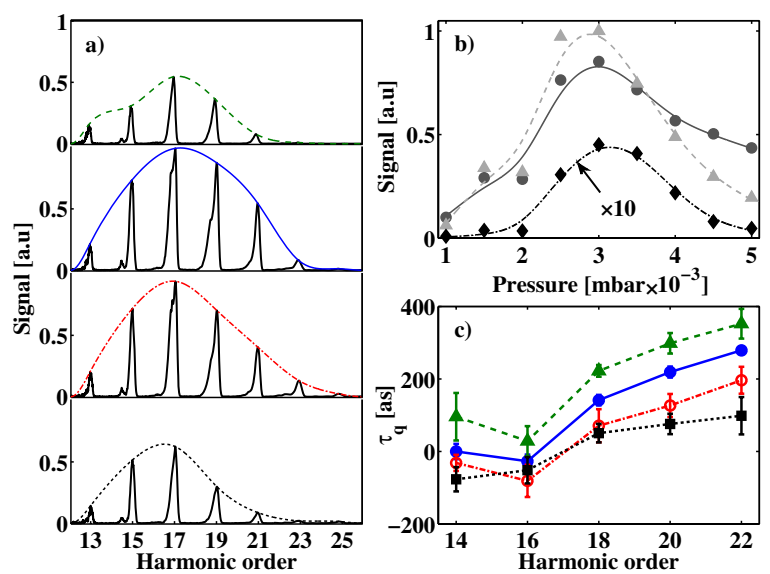

Fig. 2. Summary of the experimental results. (a) Photoelectron spectra from Ar atoms, ionized by a train of XUV pulses generated at generation pressures corresponding to $1.5 \times 10^{-3}$, $2.5 \times 10^{-3}, 3.5 \times 10^{-3}$, and $4.5 \times 10^{-3}$ mbar background pressure. An estimate of the spectral envelope, by interpolation between the peak values, is indicated. (b) Spectral amplitudes of harmonics 15 (solid circles), 19 (triangles) and 23 (diamonds, $\times 10$ ) as a function of pressure. (c) Relative group delays at the same pressures as in (a). The line types and colors match those in (a). The group delay at sideband order 14 at the pressure $2.5 \times 10^{-3}$ mbar has been arbitrarily set to zero.

corrected for the absorption cross section to reflect the spectral properties of the pulse train. In Fig. $2(\mathrm{~b})$ the spectral amplitudes of harmonics 15,19 , and 23 are plotted as a function of the background pressure. High-order harmonics exhibit a greater sensitivity to a pressure change than low-orders. This leads to a bandwidth narrowing and a shift of the spectral envelope toward lower photon energies with increasing pressure. Finally, Fig. 2(c) shows the spectrally-resolved group delay extracted from the sideband oscillations, in the energy range $22-34 \mathrm{eV}$. The data points were obtained by alternating measurements at a particular pressure and a reference pressure of $2.5 \times 10^{-3}$ mbar. The error bars were estimated by considering the variation in five such consecutive measurements. A few important features can be observed. For a given pressure, the group delay increases with harmonic order, meaning that high-order harmonics are delayed relative to low orders (positive chirp). This behavior indicates that the harmonics are dominantly generated from short electron trajectories [5,9]. The $\mathrm{Al}$ filter exhibit negative group delay dispersion in this spectral range and partly compensates for the chirp. The anomalous behavior of sideband 16 can be attributed to the $26.6 \mathrm{eV}, 3 \mathrm{~s}^{2} 3 \mathrm{p}^{6} \rightarrow 3 \mathrm{~s} 3 \mathrm{p}^{6} 4 \mathrm{p}$ resonant transition to an autoionizing state [10], affecting the phase of the two-photon ionization process. Finally, over the entire spectral range there is a negative shift of the group delay with increasing generation pressure. Understanding this effect is the focus of this Letter.

We rewrite $\tau$ as $\phi_{p} / \omega=\left[(q+1) \phi_{p}-(q-1) \phi_{p}\right] / 2 \omega$, where $\phi_{p}$ is the (variable) phase of the probe field. Since $\phi_{p}$ is related to the phase of the pump field, $\phi_{1}$, by a constant, $\delta$, which does not depend on the generating conditions and in particular not on the pressure in the generating medium, we replace $\phi_{p}$ by $\phi_{1}$ and Eq. (1) becomes 


$$
S(\tau, q)=A+B \cos \left(\Delta \phi_{q-1}-\Delta \phi_{q+1}+2 \delta\right),
$$

where $\Delta \phi_{q}=\phi_{q}-q \phi_{1}$ is the phase mismatch, i.e., the relative phase between the generated field and the polarization of the medium at the same frequency $(q \omega)$. The phase mismatch is the sum of several contributions. It includes the phase of the atomic (microscopic) dipole moment, which is related to the accumulated time the electron spends in the continuum [5] as well as the dispersion of the metallic filter. It also includes macroscopic contributions from the upconversion process, such as dispersion in the generation medium and the Gouy phase due to focusing.

For simplicity, we initially assume that the chirp induced by the single atom response ("atto-chirp") is approximately compensated for by an adequate filter. We also assume a homogeneous medium of length, $L$, and loose focusing, so that the variation of the Gouy phase can be approximated by a linear term. The first part of the argument of the cosine function in Eq. (3) can then be approximated by $2 \omega \tau_{w}$, with

$$
\tau_{w}=\frac{\Delta \phi_{q+1}-\Delta \phi_{q-1}}{2 \omega} \approx \frac{\partial \Delta \phi_{\Omega}}{\partial \Omega} \approx \frac{\partial \phi_{\Omega}}{\partial \Omega}-\frac{\phi_{1}}{\omega} .
$$

Equation (4) has a simple physical interpretation. The right-hand side represents the frequency-resolved temporal walk-off of the attosecond pulse relative to the halfcycle of the fundamental field. In the simple case where absorption can be neglected, $\Delta \phi_{q} \approx \Delta k_{q} L / 2$, where $\Delta k_{q}$ represents the wave-vector mismatch between the generated harmonic field and the component of the nonlinear polarization of frequency $q \omega$. The harmonics are, on average, generated in the middle of the medium, which explains the factor $1 / 2.2 \tau_{w} / L$ is then the difference between the inverse of the group velocity $\left(\partial k_{\Omega} / \partial \Omega\right)$ of the attosecond pulse at frequency, $\Omega$, and the inverse of the phase velocity at the fundamental frequency $\left(k_{1} / \omega\right)$. In the more realistic case, where absorption is considered, the effective interaction length depends on frequency and the temporal walk-off cannot simply be expressed in terms of velocities, but rather delays.

Including absorption and assuming a 1D geometry $[\underline{11}, \underline{12}]$, the phase mismatch can be expressed as

$$
\Delta \phi_{q}=\Delta k_{q} \frac{L}{2}-\arctan \left[\frac{\tan \left(\Delta k_{q} \frac{L}{2}\right)}{\tanh \left(\kappa_{q} \frac{L}{2}\right)}\right]+\arctan \left[\frac{\Delta k_{q}}{\kappa_{q}}\right],
$$

where $\kappa_{q}$ is the absorption coefficient at frequency $q \omega$. Figure 3(a) shows numerical estimates of different contributions to $\Delta k_{q}$ as a function of frequency, using parameters mimicking the experiment (1\% degree of ionization, corresponding to a peak intensity of $1 \times 10^{14} \mathrm{~W} / \mathrm{cm}^{2}$, and a confocal parameter of $1.3 \mathrm{~cm}$, with the focus in the center of the $6 \mathrm{~mm}$ long cell). The Gouy phase leads to a pressure-independent positive and approximately linear term (dashed-dotted line). The wave vector mismatches due to dispersion in the neutral medium and because of the generated free electrons are shown in solid and dashed lines, respectively. The respective influences of the three contributions change with varying pressure,
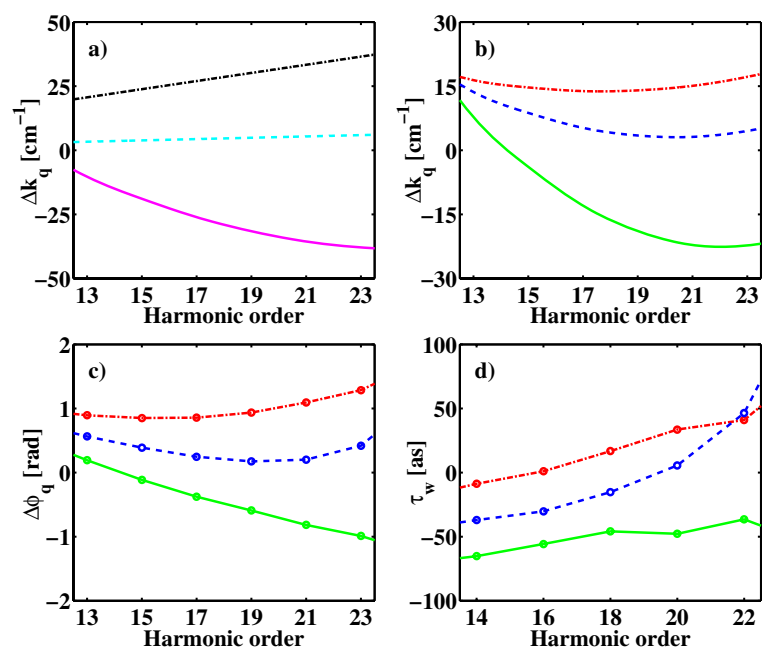

Fig. 3. (a) Contributions to the wave-vector mismatch at a gas pressure of 44 mbar: dispersion of the neutral Ar gas (solid line), dispersion of the plasma generated by the driving pulse (dashed line), and contribution from the geometrical phase (dashed-dotted line). (b) Sum of all the contributions at three different generation pressures (dashed-dotted, 27 mbar; dashed, 44 mbar; solid, 80 mbar). (c) Phase mismatch

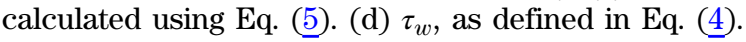

as shown in Fig. 3(b). At low pressures, $\Delta k_{q}$ is dominated by the positive Gouy phase contribution. At higher pressures, it becomes more and more influenced by neutral dispersion, leading to a characteristic decreasing curve. Figure $3(\mathrm{c})$ shows $\Delta \phi_{q}$ at three different pressures. The difference between (b) and (c) arises from absorption, which influences mainly the low harmonic orders, as well as from the coherent buildup process itself, when $\Delta k_{q} \neq$ 0 and the coherence length is less than the medium length [see Eq. (5)]. The slope of $\Delta \phi_{q}$ changes from positive when the Gouy phase is the dominant contribution to $\Delta k_{q}$, negative when, instead, dispersion is most important. The group delay therefore decreases over the whole spectral range when the pressure increases, as can be seen in Fig. 3(d).

Figure $\underline{4}$ shows the results of our 1D simulation. The model reproduces qualitatively the main experimental observations. The harmonic spectral envelope shifts to lower photon energies as the pressure increases [Fig. 4(a)]. The amplitude exhibits a maximum at the "phase matching" pressure $[\underline{13}, 14]$, where the phase mismatch is zero [Fig. 4(b)] and the phase matching pressure is almost independent of order. Finally, the group delay decreases by about 80 as from the lowest to the highest pressure [Figs. 4(b) and 4(c)]. This variation represents the temporal walk-off of the attosecond pulses relative to the fundamental field as the pressure is varied. As explained, it originates from the dispersion properties of the generating medium and, in particular, the decrease of the refractive index of argon gas for photon energies above the ionization threshold. Its magnitude depends also on the interplay of absorption and phase matching, limiting the effective generation length. The physical length of the generation medium which, under our experimental conditions, could be slightly dependent on the pressure, plays a subordinate role as the accumulated phase mismatch is determined by the absorption length 

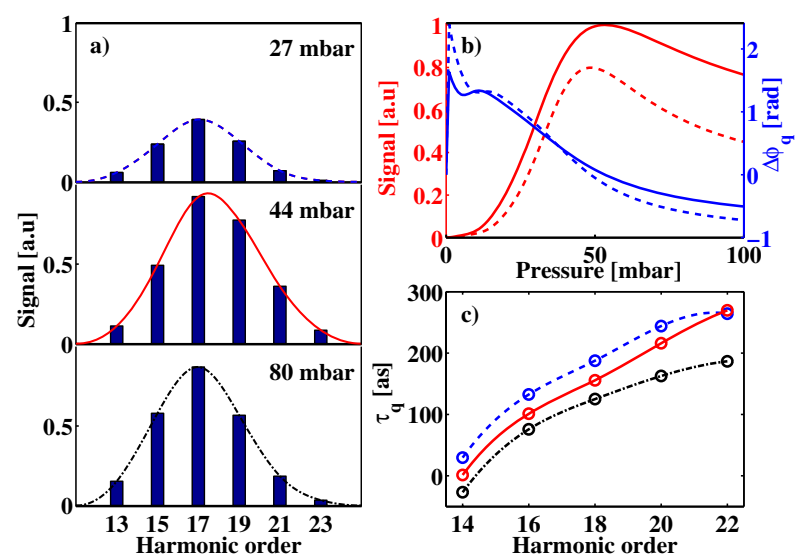

Fig. 4. 1D simulations. (a) Calculated spectral amplitudes at three different generation pressures. (b) Amplitudes (red) and $\Delta \phi$ (blue) of harmonics 17 (solid lines) and 19 (dashed lines) as a function of pressure. (c) Relative attosecond group delay, at the same pressures as in (a), including the "atto chirp" and the metallic filter dispersion.

rather than the physical length of the medium. A more quantitative description of our experimental results would require the inclusion of temporal and 3D effects, which is beyond the scope of this Letter.

In conclusion, we find that, with increasing generation pressure, the harmonic spectral envelope shifts toward lower energy and the APT acquires a negative group delay, which we interpret as a temporal walk-off. Consequently, attosecond pump-probe experiments require not only mechanical stability but also a constant pressure in the generation region. Conversely, group delay measurements, such as those presented in this work, give new insights into the generation process, highlighting the role of phase matching on the attosecond time scale.

This research was supported by the Marie Curie program ATTOFEL (ITN), the European Research Council (ALMA), the Swedish Foundation for Strategic Research, the Swedish Research Council, and the Knut and Alice Wallenberg Foundation. KV and EB acknowledge support from the Bolyai Grant of the Hungarian Academy of Sciences and the Hungarian Scientific Research Fund (OTKA project NN 107235).

\section{References}

1. P. B. Corkum, Phys. Rev. Lett. 71, 1994 (1993).

2. K. J. Schafer, B. Yang, L. F. DiMauro, and K. C. Kulander, Phys. Rev. Lett. 70, 1599 (1993).

3. P.M. Paul, E. S. Toma, P. Breger, G. Mullot, F. Augé, P. Balcou, H. G. Muller, and P. Agostini, Science 292, 1689 (2001).

4. M. Hentschel, R. Kienberger, C. Spielmann, G. A. Reider, N. Milosevic, T. Brabec, P. Corkum, U. Heinzmannß, M. Drescher, and F. Krausz, Nature 414, 509 (2001).

5. Y. Mairesse, A. de Bohan, L. J. Frasinski, H. Merdji, L. C. Dinu, P. Monchicourt, P. Breger, M. Kovačev, R. Taïeb, B. Carré, H. G. Muller, P. Agostini, and P. Salières, Science 302, 1540 (2003).

6. K. Klünder, J. M. Dahlström, M. Gisselbrecht, T. Fordell, M. Swoboda, D. Guénot, P. Johnsson, J. Caillat, J. Mauritsson, A. Maquet, R. Taïeb, and A. L'Huillier, Phys. Rev. Lett. 106, 143002 (2011).

7. L. C. Dinu, H. G. Muller, S. Kazamias, G. Mullot, F. Augé, P. Balcou, P. M. Paul, M. Kovačev, P. Breger, and P. Agostini, Phys. Rev. Lett. 91, 063901 (2003).

8. S. A. Aseyev, Y. Ni, L. J. Frasinski, H. G. Muller, and M. J. J. Vrakking, Phys. Rev. Lett. 91, 223902 (2003).

9. K. Varjú, Y. Mairesse, B. Carre, M. B. Gaarde, P. Johnsson, S. Kazamias, R. Lopez-Martens, J. Mauritsson, K. J. Schafer, P. Balcou, A. L'Huillier, and P. Salières, J. Mod. Opt. 52, 379 (2005).

10. R. P. Madden, D. L. Ederer, and K. Codling, Phys. Rev. 177, 136 (1969).

11. T. Ruchon, C. P. Hauri, K. Varjú, E. Mansten, M. Swoboda, R. López-Martens, and A. L'Huillier, New J. Phys. 10, 025027 (2008).

12. E. Constant, D. Garzella, P. Breger, E. Mével, C. Dorrer, C. L. Blanc, F. Salin, and P. Agostini, Phys. Rev. Lett. 82, 1668 (1999).

13. S. Kazamias, D. Douillet, F. Weihe, C. Valentin, A. Rousse, S. Sebban, G. Grillon, F. Augé, D. Hulin, and P. Balcou, Phys. Rev. Lett. 90, 193901 (2003).

14. P. Rudawski, C. M. Heyl, F. Brizuela, J. Schwenke, A. Persson, E. Mansten, R. Rakowski, L. Rading, F. Campi, B. Kim, P. Johnsson, and A. L'Huillier, Rev. Sci. Instrum. 84, 073103 (2013). 